\title{
XIMEHR - Modelo de interface extensível para sistemas de registro eletrônico de saúde baseados na ISO 13606
}

\section{XIMEHR - eXtensible interface model for electronic health record based on ISO13606}

\section{Modelo de interfaz extensible para sistemas de registros electrónicos de salud basado en la norma ISO 13606}

Elisa Albergaria | etuler@gmail.com

Universidade Federal de São João del-Rei, Departamento de Ciência da Computação. São João del-Rei, Brasil.

Marcello Bax | bax.ufmg@gmail.com

Universidade Federal de Minas Gerais, Escola de Ciência da Informação. Belo Horizonte, Brasil.

Raquel Prates | rprates@dcc.ufmg.br

Universidade Federal de Minas Gerais, Departamento de Ciência da Computação. Belo Horizonte, Brasil.

Zilma Reis | zilma.medicina@gmail.com

Universidade Federal de Minas Gerais, Escola de Medicina. Belo Horizonte, Brasil.

Leonardo Rocha | lcrocha@gmail.com

Universidade Federal de São João del-Rei, Departamento de Ciência da Computação. São João del-Rei, Brasil.

\section{Resumo}

O objetivo do estudo que deu origem a este artigo é propor um modelo de interface extensível (XIMEHR) para sistemas de registro eletrônico de saúde, baseados nos padrões da norma ISO 13606. A partir do conceito de Design Science, o estudo é uma resposta ao desafio à participação de usuários finais no desenvolvimento de sistemas de informação em saúde. Interfaces para prontuários eletrônicos do paciente são geradas através de um protótipo de sistema que estrutura, organiza e gerencia os conceitos clínicos. O protótipo desenvolvido foi avaliado e sua funcionalidade atendeu aos propósitos para os quais foi elaborado. Ao mesmo tempo que preserva e estrutura as informações, o modelo proposto proporcionou flexibilidade, reutilização de conceitos e permitiu a padronização do documento. Acreditamos que o produto desse estudo contribuirá para aprimorar a qualidade dos dados clínicos registrados e poderá favorecer a troca de informações entre sistemas eletrônicos utilizados na prestação de cuidado à saúde.

Palavras-chave: Ciência da informação; Gestão da informação em saúde; Informática em saúde; Registros eletrônicos de saúde; Interface usuário-computador. 


\begin{abstract}
This paper presents the results of a study which had as the main goal to propose the eXtensible interface model for electronic health record (XIMEHR) based on ISO 13606 standard. Based on the concept of Design Science, this study is a response to the challenge to the final user's participation in the development of health information systems. Interfaces for electronic health record are generated through a prototype that structures, organizes and manages clinical concepts. The prototype developed was evaluated and it fulfilled the purposes for which it was created. At the same time that the model preserves and structures the information, it also provides flexibility, reuse of the concepts and the standardization of the document. We believe that the result of this study will contribute to improve the quality of recorded clinical data and might encourage the information exchange between electronic systems used in providing health care.
\end{abstract}

Keywords: Information science; Health information management; Health information systems; Electronic health records; User-computer interface.

\title{
Resumen
}

El objetivo del estudio en que se basó este artículo es proponer un modelo de interfaz extensible (XIMEHR) para los sistemas de registro electrónico de salud, apoyado en la ISO 13606. A partir del concepto de Design Science, el estudio es una respuesta al desafío a la participación de los usuarios finales en el desarrollo de sistemas de información acerca de la salud. Interfaces con la historia clínica electrónica del paciente se generan a través de un prototipo que estructura, organiza y gestiona los conceptos clínicos. El prototipo desarrollado fue evaluado y su funcionalidad asistió a los fines para los cuales fue diseñado. Al mismo tiempo que preserva y estructura las informaciones, el modelo propuesto ha proporcionado flexibilidad, reutilización de conceptos y ha permitido la estandarización del documento. Creemos que el producto de este estudio ayudará a mejorar la calidad de los datos clínicos registrados y podrá fomentar el intercambio de informaciones entre sistemas electrónicos utilizados en la atención de salud.

Palabras clave: Ciencia de la información; Gestión de la información en salud; Sistemas de información en salud; Registros electrónicos de salud; Interfaz usuario-ordenador.

INFORMAÇÕES DO ARTIGO

Declaração de conflito de interesses: Os autores declaram não existir conflito de interesse referente à elaboração do artigo.

Fontes de financiamentos: Essa pesquisa foi parcialmente financiada pela Capes e CNPq

Considerações éticas: Não existem considerações éticas a serem feitas.

Agradecimento/Contribuições adicionais: Agradecimento para as fontes de financiamentos Capes e CNPq

Histórico do artigo: Submetido: 10.jan.2016 | Aceito: 21.jan.2016 | Publicado: 30.jun.2016

Apresentação anterior

Licença CC BY-NC atribuição não comercial. Com essa licença é permitido acessar, baixar (download), copiar, imprimir, compartilhar, reutilizar e distribuir os artigos, desde que para uso não comercial e com a citação da fonte, conferindo os devidos créditos de autoria e menção à Reciis. Nesses casos, nenhuma permissão é necessária por parte dos autores ou dos editores. 


\section{Introdução}

O registro de informações em formato eletrônico no domínio da saúde tem sido objeto de estudo e de atenção, tamanha é sua importância para o cuidado da saúde das pessoas. Há inúmeros benefícios já percebidos para a gestão da atenção às condições crônicas e agudas, quando estas informações são adequadamente documentadas, organizadas e compartilhadas. É inegável o potencial transformador da informação clínica segura e disponível no momento oportuno em que as decisões clínicas são tomadas, seja no contexto individual ou de populações ${ }^{1,2,3}$. O formato de registro conhecido como Prontuário Eletrônico do Paciente (PEP) consiste em um repositório no qual informações clínicas e administrativas, ao longo da vida de um indivíduo, ficam armazenadas ${ }^{4}$. Porém, o PEP envolve frequentemente apenas um estabelecimento de saúde e, por vezes, um único software de apoio à gestão das informações ${ }^{5}$. Para que diversas unidades de atendimentos, nos vários níveis de atenção e complexidade assistencial possam compartilhar a história clínica contida nos PEP, um novo conceito mais abrangente foi introduzido. Assim, surgiu o Registro Eletrônico de Saúde (RES) ou Electronic Health Record (EHR), em que o desafio é padronizar o formato e o significado dos dados de saúde e disponibilizá-los de forma integrada ao longo da existência ${ }^{6}$. Esse repositório longitudinal de dados é formado a partir de eventos ocorridos, ao longo do tempo, em múltiplas organizações de saúde.

Entretanto, desenvolver sistemas na área de saúde é desafiador. Armazenar informações clínicas de forma flexível, estruturada e padronizada não é trivial. Há uma enorme variedade de tipos de registros (conceitos) envolvidos nos encontros clínicos: resultantes de exames, diagnósticos, prescrições etc. E a necessidade crescente de se registrar dados sobre saúdetem evoluído com os avanços da medicina. Em consequência, requisitos demandados dos sistemas envolvem flexibilidade na criação e reutilização adaptativa de conceitos. Essas novas exigências envolvem também estruturação dos dados, reduzindo a liberalidade do texto livre em troca de um padrão que permita a recuperação de informação (por exemplo, quantos procedimentos do tipo $z$ foram realizados no paciente $y$ ?). Há ainda a complexa questão de se pactuar os padrões, uma vez que diversos locais devem armazenar informações com a mesma estrutura e significado para que possam ser compartilhadas entre os sistemas de informação.

Vários são os padrões que orientam a construção de sistemas eletrônicos de saúde voltados para troca de informações. A norma ISO13606 ${ }^{i}$ propõe a modelagem em dois níveis: o nível de conhecimento e o nível de informação ${ }^{7}$. O nível de informação, chamado de modelo de referência, define como os dados clínicos serão armazenados. Já o nível de conhecimento, composto pelos chamados arquétipos, envolve a modelagem clínica dos conceitos do domínio, idealmente feita pelos profissionais da saúde ou com a sua participação ativa $^{8}$. O objetivo desse tipo de modelagem é desvincular o modelo dos conceitos clínicos do modelo dos dados que instanciam os conceitos. Isso visa à criação de sistemas mais flexíveis, capazes de lidar com a complexidade dos modelos em saúde e resistir às alterações conceituais do domínio.

Buscando conceber sistemas de RES baseados no padrão citado, mas que sejam ao mesmo tempo flexíveis e estruturados, esta pesquisa que fundamenta este artigo propõe um modelo de interface extensível baseado em princípios da engenharia semiótica no contexto da interação humano-computador (IHC). A IHC não abrange apenas interfaces, mas todos os aspectos relacionados à interação entre pessoas e sistemas computacionais ${ }^{9,10}$. A engenharia semiótica (ES) caracteriza a interação humano-computador como um caso particular de comunicação humana mediada por sistemas computacionais ${ }^{11}$. Um protótipo validará o modelo, conforme orienta o método da Design Science Research ${ }^{12}$.

i http://www.en13606.org/the-ceniso-en13606-standard 


\section{Caracterizando o problema}

Atualmente, para que os profissionais da saúde consigam atuar ativamente na modelagem dos conceitos clínicos, eles precisam utilizar ferramentas computacionais específicas, que normalmente exigem conhecimento avançado em informática e no padrão adotado ${ }^{13,14}$. Isto tem sido um impedimento frequente para a participação mais efetiva desses profissionais, ficando a modelagem muitas vezes sem as contribuições indispensáveis daqueles que dominam os conceitos clínicos e que serão os futuros utilizadores dos sistemas. Neste contexto, vários são os sistemas desenvolvidos, por vezes impostos aos usuários finais, que apresentam dificuldades de adesão e utilização ${ }^{15,16}$.

Além disso, em sua maioria, os sistemas existentes são rígidos no sentido de não permitirem que os usuários façam customizações conceituais e estruturais, o que facilitaria a sua prática assistencial. Afinal, as especialidades médicas são inúmeras, o conjunto de profissionais de saúde que assistem aos pacientes é grande e seus perfis são diferentes, com demandas distintas.

Há soluções que permitem flexibilidade (reuso e até colaboração), mas que perdem em padronização (por exemplo, Practice Fusion ${ }^{i i}$. Existem sistemas como uma certa flexibilidade, mas que, sem a persistência estruturada dos conceitos, não permitem gerar informações por consultas e relatórios, nem promovem a interoperabilidade com outros sistemas (papel da padronização). Por outro lado, as soluções em que persistem a estrutura não são flexíveis para acomodar diferenças conceituais entre especialidades ou perfis diferentes na mesma especialidade (por exemplo, SisMateriii). Não existe hoje uma solução que apresente três características essenciais que atendam às necessidades dessa área e que contemple um padrão para troca de informações: flexibilidade, padronização e estrutura.

\section{Metodologia}

Simon $^{12}$ apresenta o conceito de Design Science, destacando a importância de uma ciência que reflete sobre artefatos para resolver problemas. Nesse contexto, Wieringa ${ }^{17}$ distingue dialeticamente problemas práticos e teóricos, sem separá-los. Um problema prático é a diferença que há entre a forma como o mundo é vivenciado pelas pessoas e como elas gostariam que fosse. Todo problema prático envolve sub problemas teóricos e práticos, de forma imbricada.

A pesquisa segue essa visão, que procura mostrar como gerar conhecimento teórico a partir da resolução de um problema prático, associando rigor e relevância. Ela é parte de um projeto maior, pautado no seguinte desafio: como possibilitar a interação de usuários finais, profissionais da saúde, em prontuários eletrônicos baseados na norma ISO 13606, permitindo que os mesmos possam personalizar sua interface mantendo estrutura e padronização dos sistemas?

Para atingir o objetivo proposto, pelos passos da Design Science Research, foi elaborado um modelo de interface extensível, o XIMEHR (eXtensible Interface Model for Electronic Health Record) para sistemas de registro eletrônico de saúde e foi desenvolvido um protótipo para validar o modelo proposto. Durante a construção do modelo e do protótipo foi feito o esforço consciente de gerar relatos conceituais teóricos fundamentados na literatura científica da área e capazes de retroalimentar essas bases de conhecimento. Este artigo é um desses relatos.

ii http://www.practicefusion.com/

iii http://sismater.hc.ufmg.br/ 


\section{O modelo XIMEHR}

Com vistas a inovar no campo dos sistemas PEP/RES, buscou-se criar um modelo que suportasse a geração de interfaces que oferecem três propriedades importantes para esses sistemas: flexibilidade, estruturação e padronização. Nesse contexto, flexibilidade permite adaptação e reuso de conceitos do domínio. Já estruturação permite a recuperação avançada de informação, via consultas sofisticadas que exploram as relações estruturais entre os dados. Ou seja, buscam-se interfaces que, ao mesmo tempo, possibilitem a customização e reuso de conceitos (flexibilidade na camada de apresentação) e a sua persistência de forma estruturada (estruturação na camada de dados). A padronização é a propriedade que facilita a interoperabilidade com outros sistemas.

O modelo XIMEHR (eXtensible Interface Model for Electronic Health Record) cria uma camada de abstração dos conceitos envolvidos no padrão da norma ISO 13606, permitindo que os usuários possam reutilizar, customizar e compartilhar conceitos e documentos, persistindo os dados de forma padronizada. No vocabulário da norma ISO13606, isso equivale a dizer que o usuário, ao personalizar seu ambiente, estará criando, editando e compartilhando arquétipos (nível do conhecimento).

Utilizando o modelo, pode-se conceber sistemas em que o usuário tem a liberdade de realizar customizações da interface, personalizando-a de acordo com suas necessidades e especialidade, mas conseguindo ainda compartilhar informações com outros profissionais. Na Figura 1 é possível visualizar o propósito do modelo. E nesse contexto, o modelo visa atuar no seguinte cenário:

Antônio é um médico que não consegue encontrar um sistema computacional que seja adequado ao seu trabalho diário. Ele gostaria que fosse possivel fazer adaptações no mesmo para que o sistema lhe atendesse de maneira mais eficiente e eficaz, mas não tem conhecimentos suficientes em computação e não está disposto a adquiri-los. Assim, Antônio gostaria de definir melhor ou até adaptar os documentos, conceitos e fluxos que estão presentes no sistema. Ele também pretende extrair informações por meio de relatórios detalhados de suas atividades e utilizar o sistema nos vários níveis de atendimento que realiza (primário, secundário e terciário). Além disso, ele gostaria de ter a possibilidade de importar ou exportar informações dos pacientes de outros sistemas.

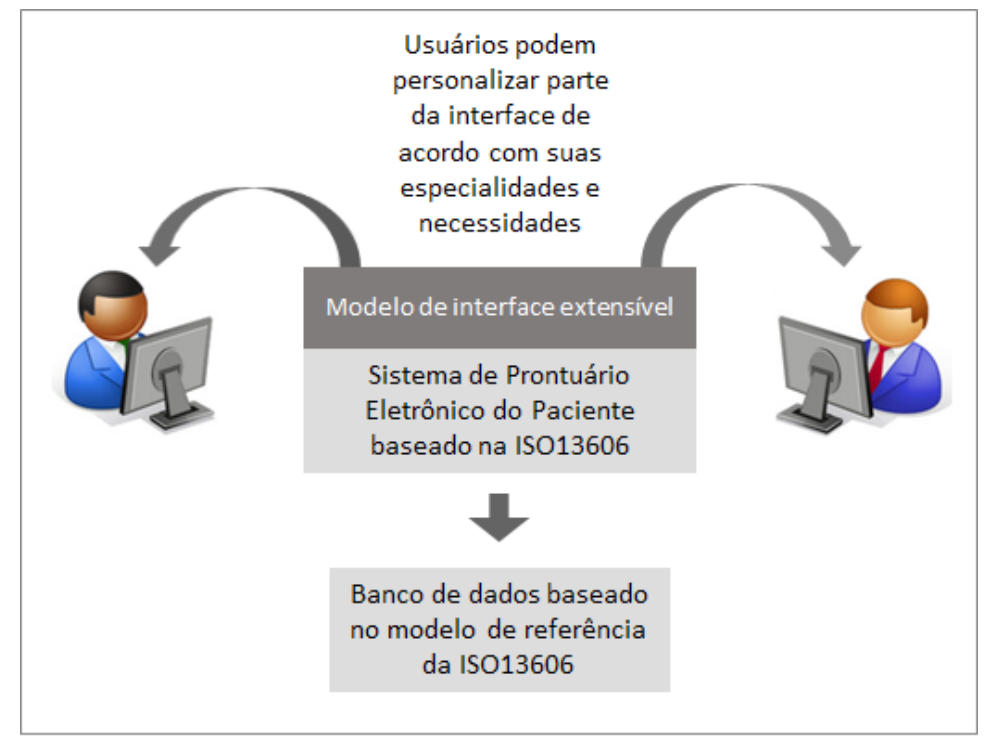

Figura 1 - Propósito do modelo XIMEHR

Fonte: Elaborado pelos autores 
Em sistemas PEP/RES desenvolvidos com o modelo proposto, não é necessário que o usuário tenha conhecimentos avançados em computação nem sobre o padrão adotado (norma ISO 13606) para que possa com eles interagir. A ideia do modelo é permitir que os dois níveis propostos pelo padrão estejam presentes, tanto o nível de informação (modelo de referência) quanto o nível de conhecimento (arquétipos). A arquitetura do modelo pode ser vista na Figura 2.

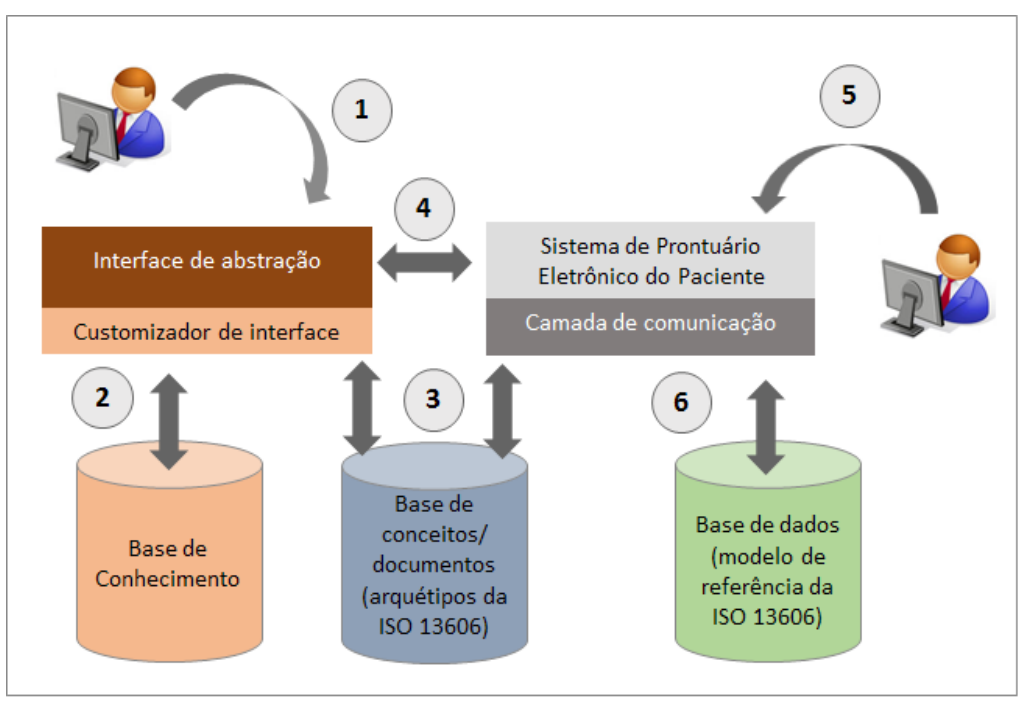

Figura 2 - Componentes e etapas de interação com o modelo XIMEHR Fonte: Elaborado pelos autores

O modelo possui três componentes: linguagem visual de interação (subdividida em interface de abstração, customizador e base de conhecimento), que permite a flexibilidade de modelagem dos conceitos; a base de conceitos/documentos, que permite a estruturação dos conceitos; e a base de dados, que permite a interoperabilidade pela transmissão e recepção de dados de outros sistemas no formato do modelo de referência ISO13606.

A primeira etapa (1), apresentada na Figura 2, consiste em permitir ao usuário configurar/personalizar seu ambiente de trabalho. Essa etapa já pode ser realizada pelo especialista em saúde, que interagindo com a interface de abstração consegue realizar suas customizações no sistema. Durante essa etapa, a base de conhecimentos é consultada (2), de forma a criar as abstrações necessárias, mantendo a ligação com os conceitos propostos pela norma ISO13606. Na base de conhecimento estão presentes as ligações entre os elementos da interface, os níveis de informação e conhecimento. Ainda durante essa criação, a base de conceitos e documentos é criada ou editada (3), visto que estes podem ser compartilhados entre os diversos especialistas de domínio e exportados para outros sistemas, se necessário. Ao finalizar essa etapa, o ambiente de interação é construído de forma flexível, mas mantendo o sistema de modo padronizado e estruturado (4). Com isso, o usuário pode interagir com o sistema, de acordo com suas necessidades de registro clínico, sem se preocupar com os conceitos de informática envolvidos (5). Os dados gerados dessa interação são armazenados numa base de dados modelada de acordo com o modelo de referência proposto pela norma ISO13606 (6). A seguir cada componente do modelo é descrito de forma mais detalhada.

\section{Linguagem visual de interação}

A linguagem visual de interação é responsável por permitir a flexibilidade proposta pelo modelo ao abstrair conceitos e permitir uma interação direta dos usuários. Ela possui três subcomponentes: a interface de abstração, o customizador da interface e a base de conhecimento. O objetivo aqui é que o customizador e a interface gerada possam ser utilizados por um especialista da saúde, sem que o mesmo precise ter 
conhecimentos avançados de computação e da ISO13606. Na construção da linguagem visual de interação, os conceitos da engenharia semiótica foram aplicados, visando a uma melhor comunicação entre o usuário e os elementos da interface.

A linguagem visual de interação criada apresenta elementos léxicos, sintático e semântico. Os sistemas computacionais apresentam uma perspectiva de manipulação de símbolos onde os elementos léxicos consistem nos elementos ou termos (que representam conceitos) envolvidos no sistema, bem como seus atributos e suas representações na interface ${ }^{18}$. A sintaxe agrupa as possíveis combinações entre os elementos válidas e adequadas para alcançar os objetivos de comunicação desejados. Finalmente, a semântica permite criar novas significações no sistema.

Os elementos léxicos da linguagem, definidos na interface de abstração foram baseados nos tipos existentes de arquétipos propostos na ISO13606 (Figura 3). Esses elementos estão descritos no Quadro 1. O modelo propõe que os principais elementos da linguagem sejam os conceitos (ENTRY) e os documentos (COMPOSITION). Os documentos consistem nos conceitos organizados visando atender às demandas de registros dos profissionais de saúde (organizados em abas - SECTION), com um certo propósito (por exemplo, o registro de um procedimento de parto). Ao se criar um conceito (um conjunto de campos), é necessário selecionar diferentes campos simples (ELEMENTs) ou tipos mais complexos, os CLUSTERs (estruturados em listas, árvores e tabelas).

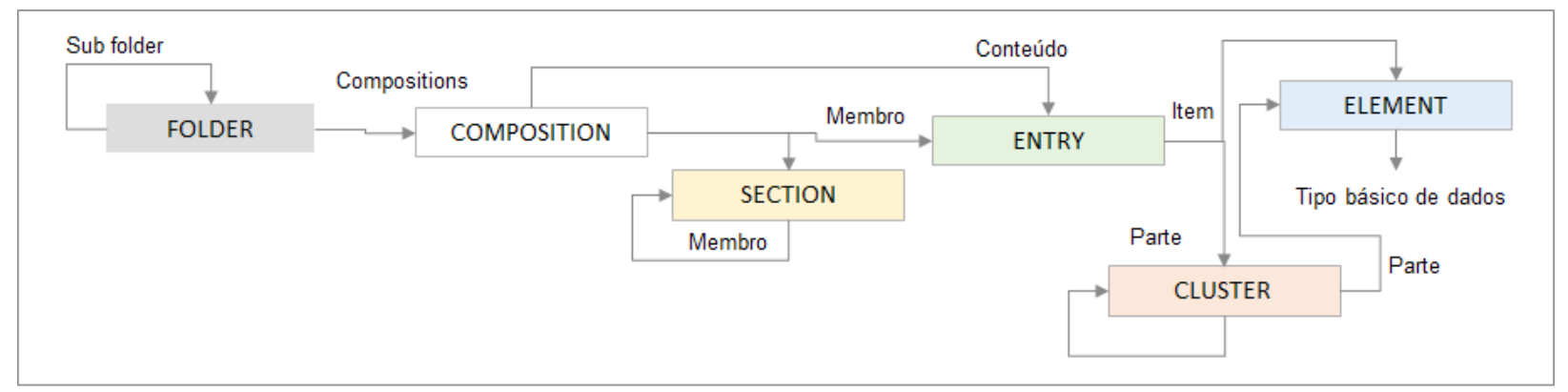

Figura 3 - Representação da informação clínica da norma ISO13606 Fonte: Elaborado pelos autores 
Quadro 1 - Elementos da linguagem de abstração

\begin{tabular}{|c|c|c|}
\hline Elementos léxicos & $\begin{array}{l}\text { Significado na norma } \\
\text { ISO13606 } \\
\text { (Modelo de referência) }\end{array}$ & Descrição \\
\hline Perfil do usuário & Folder & $\begin{array}{l}\text { Conjunto de Compositions (documentos) que } \\
\text { compõem uma especialidade médica, em um } \\
\text { ambiente de saúde. Por exemplo, é possível ter } \\
\text { um folder para "ginecologia em hospital" ou } \\
\text { "ginecologia em consultório". }\end{array}$ \\
\hline Documento & Composition & $\begin{array}{l}\text { São os documentos que compõem um PEP. } \\
\text { Cada documento consiste em um formulário } \\
\text { de entrada de dados. Podem comportar os } \\
\text { protocolos de atendimento de uma instituição } \\
\text { de saúde. }\end{array}$ \\
\hline ABAS & Section & $\begin{array}{l}\text { Consistem nas abas dos formulários, sendo } \\
\text { que cada uma delas representa uma seção do } \\
\text { documento. }\end{array}$ \\
\hline Conceito & Entry & $\begin{array}{l}\text { São os conceitos clínicos, formados por um } \\
\text { conjunto de campos estruturados, que contêm } \\
\text { os dados e metadados de tal conceito. Vários } \\
\text { conceitos formam um documento. }\end{array}$ \\
\hline $\begin{array}{l}\text { Campo composto (lista, } \\
\text { árvore, tabela) }\end{array}$ & Cluster & $\begin{array}{l}\text { Consiste em um conjunto de dados que } \\
\text { caracterizam os campos compostos. Comportam } \\
\text { tabela, lista, série temporal ou árvore de dados. }\end{array}$ \\
\hline Campo simples & Element & $\begin{array}{l}\text { Consiste em um campo simples do formulário, onde } \\
\text { é definido o tipo de dado que pode ser inserido, sua } \\
\text { cardinalidade, sua obrigatoriedade etc. }\end{array}$ \\
\hline
\end{tabular}

Fonte: Elaborado pelos autores

O customizador de interface consiste nos recursos disponíveis para que o usuário possa personalizar sua interface, funcionando com a sintaxe da linguagem visual de interação que permite definir como os elementos léxicos são combinados. Esses recursos devem permitir que o usuário possa gerenciar documentos e conceitos, além de alterar seu perfil e realizar outras personalizações necessárias.

A base de conhecimento, subcomponente da linguagem visual de interação, aborda de forma mais detalhada os conceitos envolvidos na norma ISO13606, armazenando as relações, conceitos e os atributos dos elementos léxicos criados, considerando a correspondência com a norma. Ela funciona como a semântica da linguagem, definindo o significado dos elementos. O papel da base de conhecimento é estabelecer a relação entre os elementos da interface criada com os níveis de conhecimento e de informação da norma ISO13606. Como exemplo para ilustrar as informações presentes na base de conhecimento, é possível visualizar na Figura 4 a relação entre os tipos primitivos presentes no modelo de referência da ISO13606 (apresentados em cinza, por exemplo, SIMPLE_TEXT), que foram mapeados nos tipos que devem ser apresentados aos usuários (na cor verde, por exemplo, TEXTO). Na Figura 4 também estão representados 
conceitos presentes no pacote estrutural de arquétipos que devem estar presentes na interface, abstraídos para os usuários. O pacote estrutural proposto na norma ISO13606 inclui uma descrição e uma ontologia. Assim, algumas informações como: nome do "arquétipo", data de criação, idioma e os termos envolvidos devem ser definidos no momento em que conceitos e documentos são criados. Além disso, ao se criar um conceito, são criados vários campos, simples ou complexos (ELEMENTS e CLUSTER) e, em cada criação, a lista e a descrição de termos relacionados ao campo devem ser atualizadas (ontologia).

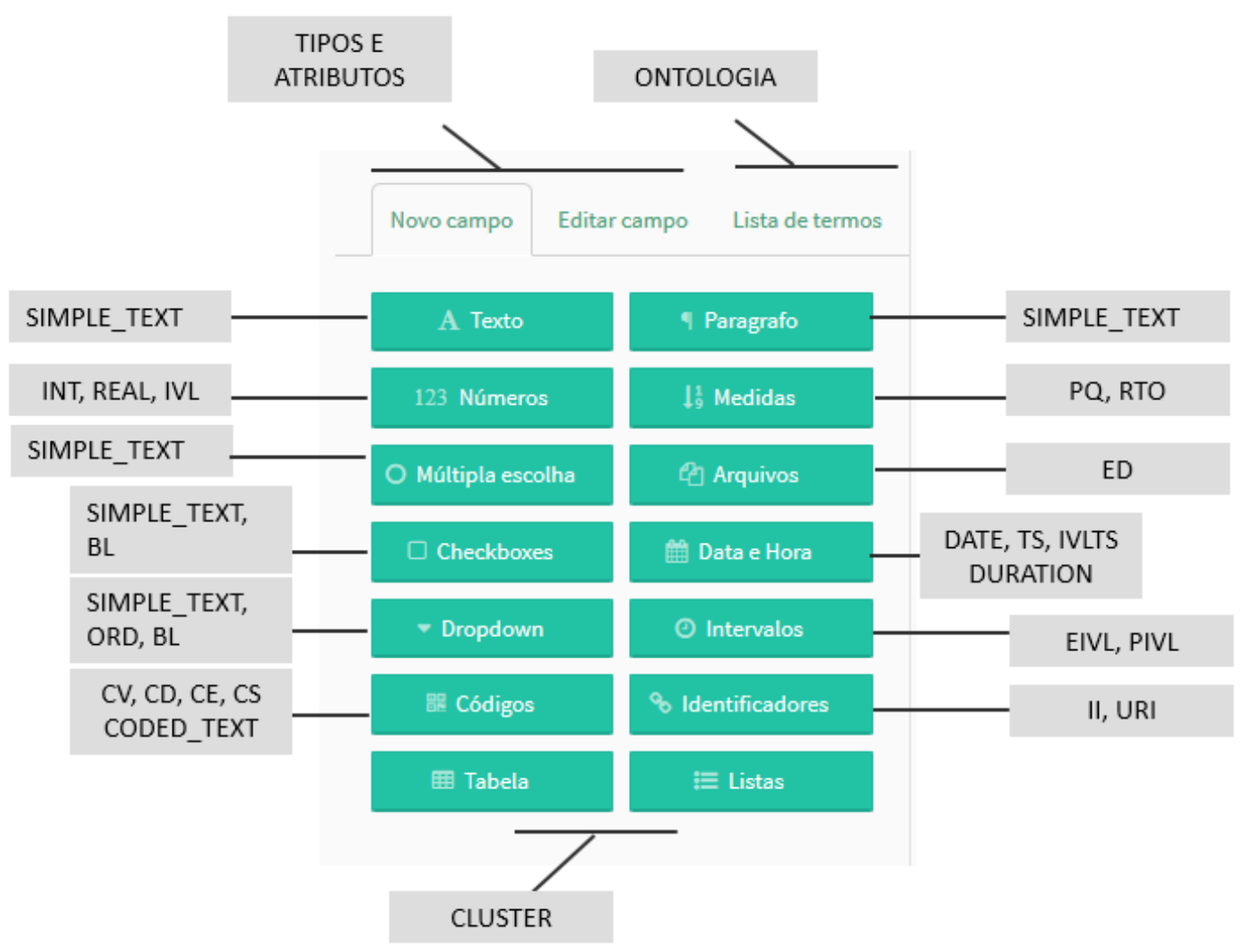

Figura 4 - Tipos simples do modelo de referência da ISO 13606 Fonte: Elaborado pelos autores

\section{Banco de conceitos/documentos}

O banco de conceitos/documentos é responsável pela característica de estruturação do modelo, no qual são armazenados conceitos e documentos seguindo o nível de conhecimento proposto na norma ISO13606, ou seja, em forma de arquétipos. Eles podem ser desenvolvidos diretamente por profissionais da saúde, permitindo que sejam gerenciados e compartilhados, sem que os usuários tenham necessariamente conhecimento específico sobre o padrão informacional adotado.

Na proposta do modelo XIMEHR, o usuário pode gerenciar e compartilhar diretamente dois tipos de arquétipos: os documentos (COMPOSITION) e os conceitos (ENTRY). Além disso, o usuário pode redefinir seu perfil, alterando o tipo FOLDER que deseja criar. Um mesmo profissional pode ter mais de uma especialidade e assim propor documentos que podem estar em folders distintos. Por exemplo, um profissional pode atuar em pediatria e, simultaneamente, no contexto medicina de família, criando documentos para as duas subáreas da saúde. Além disso, um documento (COMPOSITION) pode ser de uso transversal, ou seja, fazer parte de vários contextos de utilização no cuidado em saúde, como por exemplo um documento padronizado de alta após internação hospitalar pode ser usado em inúmeros contextos clínicos ${ }^{19,20}$. 


\section{Banco de dados}

O banco de dados permite a padronização e contribui para a interoperabilidade entre sistemas informatizados de saúde. Ele consiste no repositório de dados em si, que correspondem às informações fornecidas pelos usuários. Nesse repositório estarão armazenadas as informações sobre a saúde dos pacientes. Pretende-se que tais informações sejam armazenadas utilizando-se o modelo de referência da norma ISO13606. Vale ressaltar que alguns estudos vêm sendo realizados no sentido de analisar como esses dados devem ser armazenados, como o apresentado por Pessanha e $\mathrm{Bax}^{21}$.

Com isso, é possível compartilhar dados clínicos entre os profissionais de saúde de um mesmo paciente, simultaneamente ou em contextos distintos da assistência ao paciente. Há uma grande vantagem no reaproveitamento de dados sobre a saúde em ambientes diferentes. Por exemplo, a informação sobre o grupo sanguíneo de um indivíduo ou as reações adversas a um medicamento podem ser muito úteis e oportunas em um atendimento hospitalar ou ambulatorial e deveriam ser compartilhadas com outros profissionais de saúde. Em diferentes locais de atendimento, em sistemas de informação distintos e por profissionais de saúde autorizados, dados de saúde poderão ser compartilhados, visualizados e atualizados através do modelo XIMEHR.

\section{Protótipo e validação}

De forma a validar o modelo XIMEHR, um protótipo foi desenvolvido baseado no framework Bootstrapiv ${ }^{\text {. }}$ Utilizando o protótipo, foi feita a modelagem do SSsumário de alta de internação obstétrica no Hospital das Clinicas da UFMG ${ }^{19,20}$. Dessa forma, os conceitos e campos apresentados nas interfaces simulam a interação de usuários realizando essa modelagem. De forma a ilustrar os componentes do modelo, a seguir serão apresentadas algumas telas do protótipo referenciando os componentes do XIMEHR.

Na Figura 5 são apresentados os elementos léxicos definidos na interface de abstração. O customizador refere-se aos recursos disponíveis para que o usuário possa personalizar sua interface e gerenciar documentos e conceitos. No protótipo, esses recursos estão disponíveis no menu "Gerenciar" e permite: customizar o perfil dos usuários e estrutura dos dados (FOLDER), compartilhar e gerenciar (criar, editar, deletar) documentos; compartilhar e gerenciar (criar, editar, deletar) conceitos.

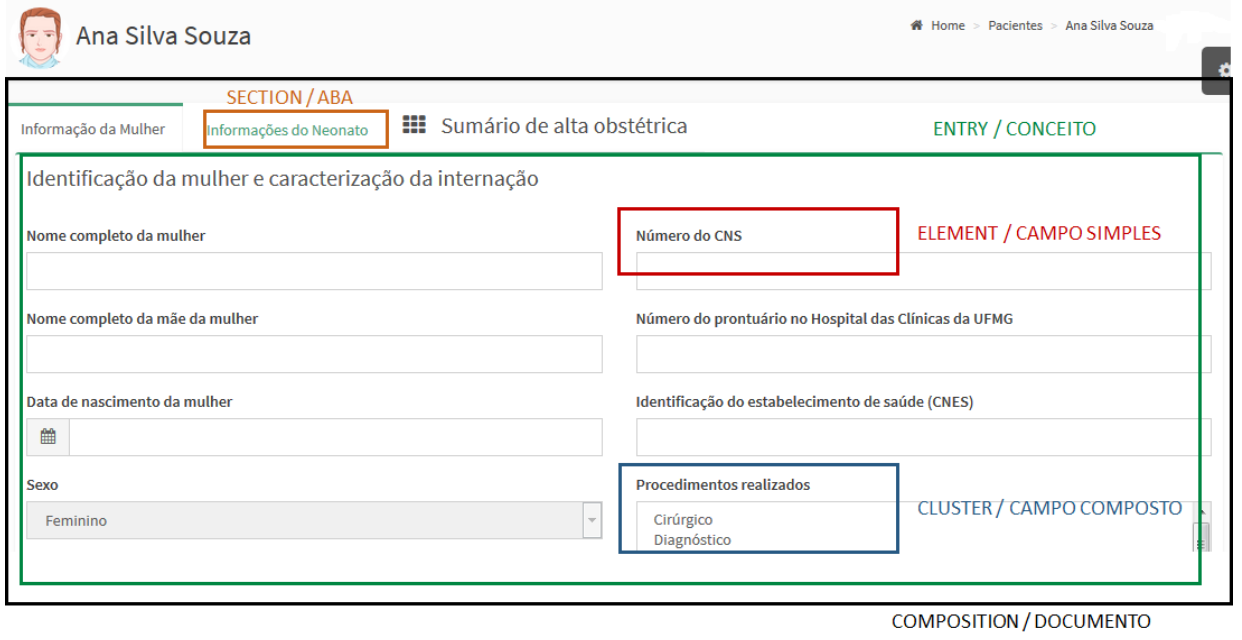

Figura 5 - Representação gráfica dos elementos léxicos Fonte: Elaborado pelos autores

iv http://getbootstrap.com/ 
O recurso apresentado no protótipo para criar conceitos permite que o usuário selecione os campos de diferentes tipos, como apresentado na Figura 6. Ao selecionar um campo, o usuário deve indicar o nome do conceito, além de informar outros dados como obrigatoriedade (editar campo) e definir os termos relacionados ao conceito que está sendo criado (lista de termos).

As relações entre os tipos de campos apresentados e os descritos na ISO13606 ficam armazenadas na base de conhecimento, como ilustrado na Figura 4. Os campos criados aparecem na parte direita da tela, como "Nome completo da mulher", de acordo com o tipo escolhido.

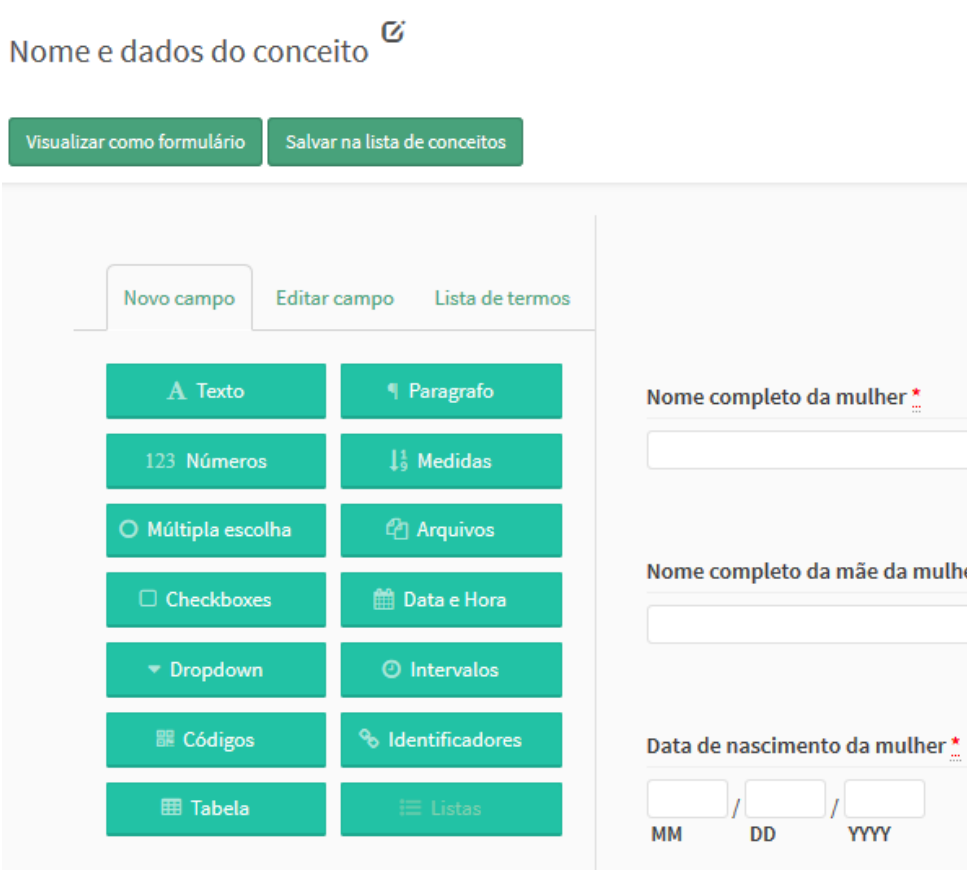

Figura 6 - Recursos para criação de conceitos Fonte: Protótipo XIMEHR

A Figura 7 apresenta a tela que permite que o usuário possa criar um novo documento, organizando os conceitos em diferentes abas (SECTIONS) que podem ser criadas pelo usuário. Para isso, basta que o usuário arraste e solte os conceitos, podendo depois organizar e visualizar o documento como um formulário.

\section{Novo Documento}

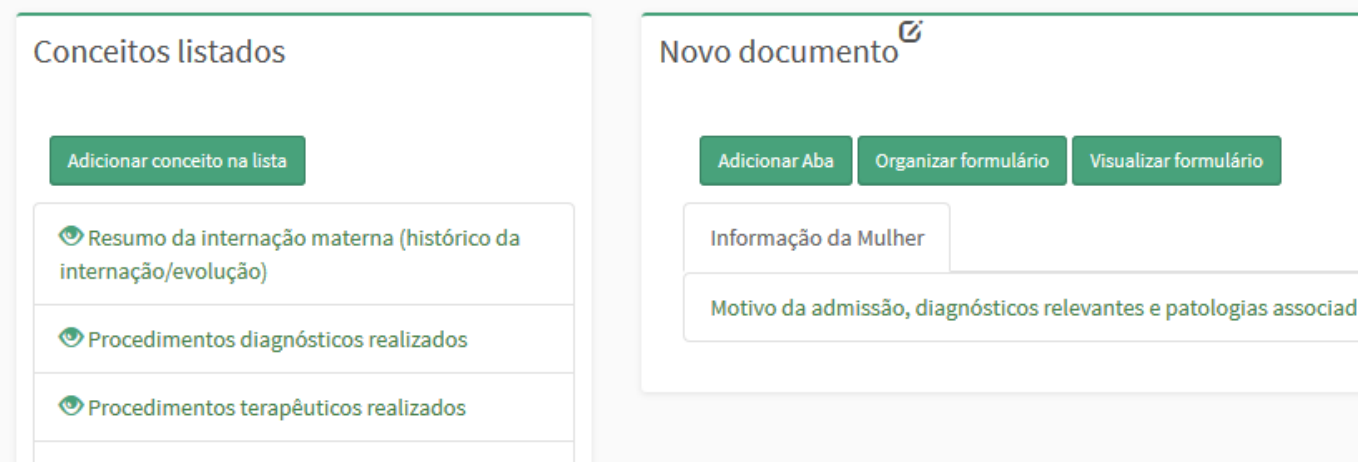

Figura 7 - Interface para criação de novo documento Fonte: Protótipo XIMEHR 
O modelo também permite que os usuários possam gerenciar seus próprios documentos de interesse, como mostra a tela apresentada na Figura 8. Além disso, o usuário pode compartilhar um documento ou obter algum que tenha sido compartilhado, fazer uma cópia do mesmo e utilizar em seu ambiente de trabalho.

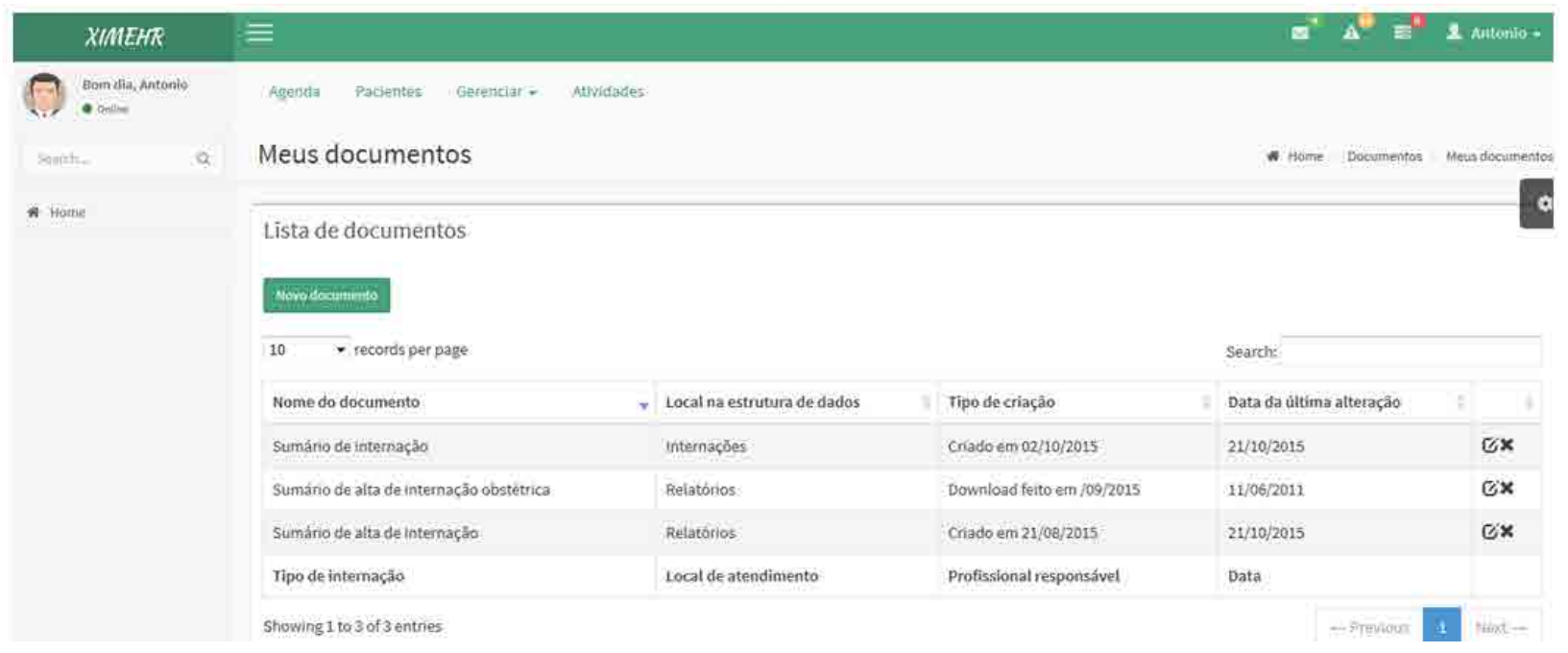

Figura 8 - Tela do protótipo: gerenciamento de documentos Fonte: Protótipo XIMEHR

Da mesma forma, os usuários podem gerenciar e compartilhar conceitos, como mostra a Figura 9. O profissional pode visualizar conceitos de uma determinada especialidade, criar uma cópia desse conceito e usá-lo ou editá-lo. Posteriormente, ele pode usar esse conceito para compor o documento que deseja.

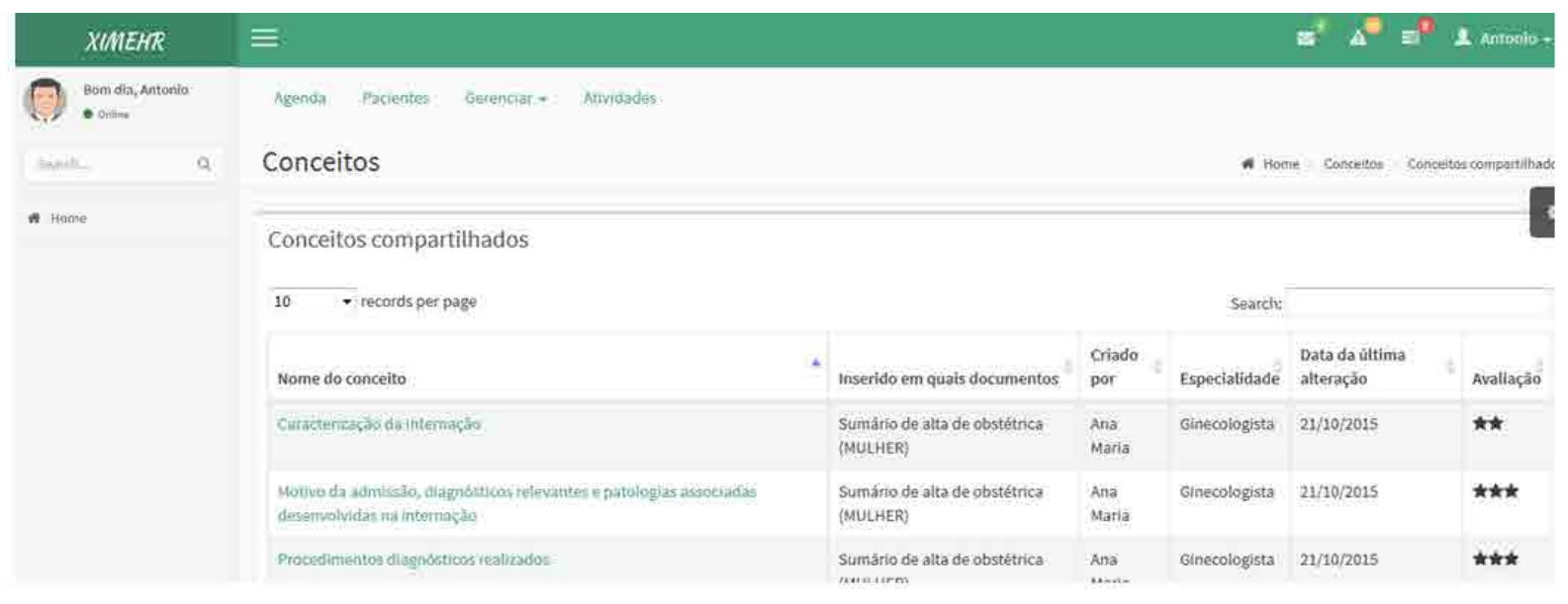

Figura 9 - Tela de compartilhamento de conceitos Fonte: Protótipo XIMEHR

\section{Conclusão}

O XIMEHR é um modelo que suporta a geração de interfaces em sistemas PEP/RES oferecendo três propriedades importantes para esses sistemas: flexibilidade, estruturação e padronização. A flexibilidade permite que os próprios profissionais de saúde possam gerenciar seus conceitos e documentos, criando interfaces personalizadas. A estruturação e a padronização permitem consultar e compartilhar informações dos pacientes em diferentes ambientes e por profissionais de saúde (o que exige algum grau de interoperabilidade). 
O modelo é baseado na norma ISO13606, que adota a modelagem de dois níveis, separando as classes usadas para armazenagem dos dados, dos conceitos clínicos gerenciados pelos profissionais da saúde. Cabe ressaltar que uma pesquisa preliminar visando à validação da proposta do modelo aqui apresentado foi realizada com profissionais da saúde e obteve uma visão positiva quanto ao seu uso ${ }^{22}$. De forma a validar o modelo, o protótipo foi desenvolvido e a modelagem do sumário de alta obstétrica realizada, atendendo aos objetivos propostos.

Uma das propostas de trabalhos futuros consiste em validar o modelo e protótipo em testes com usuários finais, os profissionais da saúde.

\section{Referências}

1. Detmer, D; Bloomrosen M; Raymond B, et al. Integrated personal health records: transformative tools for consumer-centric care. BMC Med Inform Decis Mak 2008;8:45.

2. Hillestad R, Bigelow J, Bower A, et al. Can electronic medical record systems transform health care? Potential health benefits, savings, and costs. Health Aff (Millwood) 2005;24:1103-17

3. Paul C. T and David L. The missing link: bridging the patient-provider health information gap. Health Affairs, 24, no.5 (2005):1290-1295

4. Massad, E.; Marin, H. F.; Azevedo, R. S. O prontuário eletrônico do paciente na assistência, informação e conhecimento médico. Ed. do Autor. São Paulo, 2003.

5. Santos, M. R.(2011);. Sistema de registro eletrônico de saúde baseado na norma ISO 13606: aplicações na Secretaria de Estado de Saúde de Minas Gerais. PhD thesis, UFMG, Brasil.

6. ABNT ISO (International Organization for Standardization)/TR 20.514 - Informática em saúde - Registro eletrônico de saúde - Definição, escopo e contexto

7. ISO/TC251 13606 Health informatics - Electronic record communication - Part 1: Reference Model and Part 2: Archetype interchange. ISO, 2008.

8. Beale, T.; Heard, S.(2007) A archetype definitions and principles. disponível em:<http://www.openehr.org/ releases/1.0.2/architecture/am/archetype_principles.pd>

9. Albergaria, E. T. ; Bax, M. P. ; Prates, R. O. . Interação humano-computador na ciência da informação. In: XIV Encontro Nacional de Pesquisa em Ciência da Informação, 2013, Florianópolis.

10. Preece, J.; Rogers, Y.; Sharp, H.; Benyon, D.; Holland, S. e Carey, T (1994). Human-computer interaction. Addison Wesley, England.

11. de Souza, C. The semiotic engineering of human-computer interaction. MIT Press, Cambridge; 2005.

12. Simon, H. A. The sciences of the artificial. 3. ed. USA: MIT Press, 1996.

13. Albergaria, E. T.; Bax, M. P. ; Prates, R. O. ; Rocha, L. Caracterizando desafios de interação em ferramentas de modelagem de dados clínicos. In: XIV Congresso Brasileiro em Informática em Saúde, 2014, Santos - SP.

14. Albergaria, E. T.; Bax, M. P. ; Prates, R. O. ; Rocha, L C D . Caracterizando os desafios na modelagem dos dados clínicos em sistemas de RES baseados no OpenEHR. In: WIM - XIV Workshop de Informática Médica. Anais do XXXIV Congresso da Sociedade Brasileira de Computação, 2014, Brasília.

15. Clarke, M. A., Steege, L. M., Moore, J. L., Belden, J. L., Koopman, R. J., \& Kim, M. S. (2013). Addressing human computer interaction issues of electronic health record in clinical encounters. In A. Marcus (Ed.), Design, user experience, and usability: health, learning, playing, cultural, and cross-cultural user experience(pp. 381-390). Berlin, Germany: Springer.

16. Zahabi, M; Kaber, D B.; Swangnetr, M. Usability and safety in electronic medical records interface design a review of recent literature and guideline formulation. Human Factors: The Journal of the Human Factors and Ergonomics Society, p. 0018720815576827, 2015.

17. Wieringa, R. Design science as nested problem solving. In: Proceedings of the 4th international conference on design science research in information systems and technology. ACM, 2009. p. 8.

18. de Souza, C.S.e Barbosa,S.D.J.(2006).A semiotic framing for end-user development. p. 401-426, 2006. 
19. Ferreira, A. A. T. ; Reis, Z. S. N. ; Frade, S. ; Gaspar, J S ; Hadad, S. ; Cruz-Correia R J ; Santos, M.R.S . Proposição de um sumário de alta obstétrico visando a troca de informações, em padrão OpenEHR, para continuidade do cuidado materno-infantil. Medicina (Ribeirao Preto. Online), v. 47, p. 59-66, 2014.

20. Reis, Z S N ; Aguiar, R.; Rego, M. A.; Osanan, G C; Gaspar, J S . Proposta de norma ABNT: sumário de alta de internação obstétrica. 2013.

21. Pessanha, C P; Bax, M P. Implementando o prontuário eletrônico OpenEHR em sistemas gestores de conteúdo: uma aproximação. In: XVI Encontro Nacional de Pesquisa em Ciência da Informação, 2015, João Pessoa.

22. Albergaria, E. T. ; Andrade, C. C. ; Prates, R. O. ; Bax, M. P. . Criação de um modelo de interface extensível para sistemas de registro eletrônico de saúde. In: XIII ENANCIB - Encontro Nacional de Pesquisa em Ciência da Informação, 2012, Rio de Janeiro. 